\title{
Aspetti biomolecolari della malattia renale policistica (PKD)
}

\section{Scolozzi}

Estratto della Tesi di Specializzazione in Biochimica e Chimica Clinica

Università degli Studi di Pisa, Anno Accademico 1997/98

cscolozzi@hotmail.com

PKD: BiomoleculaR ASPECTS

Abstract. Autosomal dominant polycystic kidney disease (ADPKD)is the most common genetic kidney disease with an estimated prevalence of between $1: 400$ and $1: 1,000$. caused by germline mutations in $P K D 1$ (85\%) or PKD2 (15\%). The cardinal feature of ADPKD is the presence of multiple fluid-filled kidney cysts which enlarge over time. Cyst initiation and expansion is a complex process characterized by abnormalities in tubular cell proliferation, fluid secretion, extracellular matrix formation and cell polarity.

Currently, the three major treatment strategies are to: 1) reduce cAMP levels; 2) inhibit cell proliferation, and 3) reduce fluid secretion.

Key words: ADPKD, Cyst initiation, Tubular cell proliferation

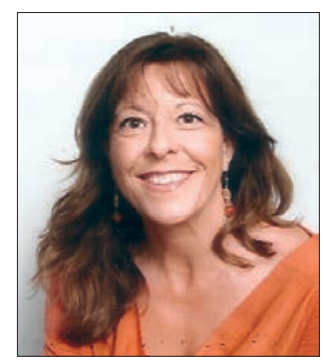

Cosima Scolozzi

\section{Introduzione}

La malattia renale policistica (PKD) è un disordine multigenico caratterizzato dall'accrescimento di cisti contenenti fluido, all'interno dei nefroni e nei dotti collettori dei reni degli individui affetti. Sono stati individuati due distinti tipi di PKD: la forma autosomica dominante (ADPKD), che si manifesta principalmente in età adulta, e la forma recessiva (ARPKD), molto più grave, che si manifesta più precocemente nel corso della vita (Gabow P.A. 1993).

Al momento si conoscono tre geni differenti, responsabili della forma dominante di questa malattia, che peraltro si manifesta anche a livello di fegato, pancreas, intestino, valvole cardiache, cervello, facendo supporre che i geni di PKD siano attivi in una serie di tessuti ed organi. Il gene PKD1 è stato identificato nel 1994 (The Europ. Poyc.Kid. Dis. Cons. 1994); esso è localizzato sul cromosoma 16 (16p 13.3) e causa l'85-90\% dei casi di ADPKD; misura circa $54 \mathrm{~kb}$ ed è trascritto in un mRNA di circa $14 \mathrm{~kb}$, che si traduce in una proteina piuttosto grande (4303 aminoacidi), dal peso di circa $460 \mathrm{kd}$, denominata policistina 1 (The Int. Pol. Kid. Dis.Cons. 1995).

La restante parte dei casi di ADPKD è dovuta a muta- zioni del gene PKD2, identificato nel 1996 e che si trova nel braccio lungo del cromosoma 4 (4q 22); questo gene misura circa $68 \mathrm{~kb}$, viene trascritto in un mRNA di 5,4 $\mathrm{kb}$, che viene poi tradotto in una proteina di $968 \mathrm{ami}-$ noacidi, dal peso di circa $110 \mathrm{kd}$, chiamata policistina 2 (Mochizuki T. et al 1996).

Inoltre esiste il gene PKHD1, che causa la forma recessiva ARPKD, mappato sul cromosoma 6 (6p 21-p22) (Reeders S.T. 1992).

\section{Aspetti biomolecolari di ADPKD}

Una serie di anomalie, apparentemente non in relazione tra loro, sono state evidenziate in cellule e tessuti provenienti da soggetti affetti da questa disfunzione, ma anche da altre forme di patologie renali dovute a cisti. Le più documentate sono le anomalie nella composizione delle membrane basali tubulari, la proliferazione delle cellule epiteliali tubulari e l'inversione della polarità delle membrane cellulari.

Finora sono state identificate 20 differenti mutazioni del gene PKD1; la varietà delle mutazioni è un fattore che contribuisce all'eterogeneità della malattia. Inoltre la ricerca delle varie mutazioni è complicata dal fatto che molti geni sono ripetuti.

Molte delle mutazioni che inattivano PKD1 e PKD2 
portano a codoni nonsenso, frameshifts, difetti di spicing, delezioni, premature terminazioni della traduzione e proteine tronche (Viribay M. et al. 1997, Daniells C. et al 1998).

Un piccolo numero di mutazioni è dato da una semplice sostituzione di aminoacidi, che può causare profondi effetti sulla attività della proteina o anche la sua inattivazione (Xenophontos S. et al. 1997).

La policistina 1 è espressa nell'epitelio biliare di fegato, nel pancreas, negli epiteli del dotto cardiaco, nell'endotelio vascolare, nella muscolatura liscia, nei fibroblasti, nelle cellule endoteliali della vena ombelicale e in molti altri distretti cellulari.

Le cisti probabilmente derivano da un'espansione clonale di singole cellule (Torres V.E. 1998), come fa supporre l'osservazione che le regioni renali implicate nel processo degenerativo sono relativamente limitate.

Nei casi avanzati di ADPKD, il parenchima renale è quasi completamente sostituito da cisti; l'interstizio è molto espanso a causa dell'aumento della matrice extracellulare, dell'infiltrazione di leucociti e fibroblasti e della disposizione di materiale fibrotico.

Alcuni ricercatori hanno suggerito che ciò possa essere causato semplicemente dalla distorsione dell'architettura renale; alterazioni della perfusione sanguigna nella corteccia renale e distruzione dei complessi che concorrono alla circolazione di sangue ed urina nella midollare renale finiscono per diminuire la filtrazione glomerulare e la funzione tubulare (Calvet 1998).

Nella maggioranza dei fluidi rimossi da cisti umane o di ratto sono stati rinvenuti agonisti secretori e sono stati riscontrati livelli di AMP ciclico più elevati della norma all'interno delle cellule epiteliali (Ye M. et al. 1992 , Yamaguchi et al. 1997).

In base a queste osservazioni è stato coniato il termine CAF (cyst-activating-factor) per identificare l'attività del lipide o delle famiglie di lipidi presenti nelle cisti umane e di ratto (Grantham J.J.1997). CAF verrebbe prodotto nelle cellule epiteliali delle cisti e potrebbe promuovere il loro allargamento e l'infiammazione interstiziale; il processo comincerebbe in quei segmenti tubulari in cui le cisti hanno avuto inizio a causa di eventi mutazionali specifici.

L'epitelio delle cisti ha la capacità di assorbire e secernere $\mathrm{NaCl}$ e fluidi; nelle cisti "prossimali”, che sono usualmente isolate dal fluido afferente dai tubuli, la concentrazione degli osmoliti organici non può essere generata o mantenuta all'interno del fluido delle cisti, la cui composizione è determinata dalla competizione tra assorbimento attivo di sodio e se- crezione attiva di cloro.

Nelle cisti "distali", relativamente impermeabili, ricche di giunzioni paracellulari e che mantengono le connessioni con i segmenti tubulari afferenti, la stimolazione di cAMP da parte di AVP, secretina, VIP o dello stesso lipide secretorio delle cisti, porta all'aumento della secrezione di fluido e di cloro all'interno della cavità delle cisti, che qui si ritrova ad una concentrazione più alta di quella che ha nel liquido extracellulare (Grantham J.J. 1996 ).

Quindi si può supporre che la velocità con cui il fluido riempie le cisti sia data dal modo in cui elettroliti e osmoliti organici (aminoacidi, polipeptidi ecc.) vengono depositati all'interno delle cisti; nella maggior parte delle cisti sembra che la secrezione di cloro sia sotto il controllo del cAMP intracellulare ed è interessante notare che la diminuzione della secrezione di $\mathrm{Cl}^{-}$per vie farmacologiche può portare ad un processo di assorbimento di $\mathrm{NaCl}$ che porta al collasso delle cisti, specie quelle con una permeabilità ai soluti e alle macromolecole relativamente alta (Grantham J.J. 1996).

E stato dimostrato che PKD viene esacerbato da stati di acidosi o da bassi livelli di potassio e si hanno dimostrazioni del fatto che la somministrazione di alcali può avere un effetto benefico, in ratti Han:SPRD, usati come modello di PKD umano (Torres V.E. et al. 1994). Trattando gli stessi ratti con soluzioni di potassio citrato/acido citrico (Kcitr) fin da quando questi hanno l'età di un mese, si previene il declino della funzionalità renale, anche se non si osserva riduzione del volume o del numero delle cisti (Tanner G.A. 1998).

Si possono fare numerose ipotesi:

l'acido citrico è un substato metabolico nei reni e quindi può aumentare la produzione di energia nelle cellule renali (Elhamri M.1993);

l'effetto di alcalinizzazione del citrato può ostacolare l'acidosi metabolica che frequentemente accompagna le disfunzioni renali;

il citrato stesso è un antiossidante e si conoscono gli effetti negativi che molecole di ossidanti possono avere sulla progressione di PKD (Torres V.E. et al. 1997). Negli ultimi anni le conoscenze sulle caratteristiche molecolari e genetiche della PKD si sono ampliate e si è dato molto spazio alla ricerca.

Purtroppo il meccanismo biochimico che sta alla base di questa patologia non è ancora del tutto noto, anzi sono state formulate teorie anche contrastanti tra loro, circa l'eventuale meccanismo d'azione dei geni e delle relative proteine, le quali, con la loro struttura piuttosto complessa, complicano non poco l'individuazione di una terapia farmacologica efficace. 


\section{Riassunto}

La malattia renale policistica autosomica dominante $(\mathrm{ADPKD})$ è la più comune malattia genetica renale, con incidenza stimata di fra 1:400 e 1:1.000 e causata da mutazioni genetiche di PKD1 (85\%) o PKD2 (15\%). La caratteristica principale di ADPKD è la presenza di cisti multiple piene di liquido che si ingrandiscono nel tempo. L'espansione e l'iniziazione delle cisti è un complesso processo caratterizzato da anomalie nella proliferazione cellulare, nella secrezione di fluidi, nella formazione della matrice extracellulare e nella polarità delle cellule. Attualmente le strategie terapeutiche maggiormente indagate sono tre: 1) riduzione del livello di cAMP; 2) inibizione della proliferazione cellulare; 3 ) riduzione della secrezione di fluidi nelle cisti.

Parole chiave. ADPKD, Iniziazione cisti, Proliferazione cellule tubulari

\section{cscolozzi@hotmail.com}

\section{Bibliografia}

1. Calvet JP. Molecular genetics of polycystic kidney disease. J Nephrol 1998; 11: 24-30.

2. Daniells C, Maheshwar M, Lazarou L, Davies F, Coles G, Ravine D. Novel and recurrent mutation in the PKD1 gene

3. Hum Genet 1998; 102: 216-220.

4. Elhamri M, Martin M, Ferrier B, Baverel G. Substate uptake and utilisation by the kidney of fed and starved rats in vivo. Renal Physiol Biochem 1993; 16: 311-24.

5. Gabow PA. Autosomal dominant polycystic kidney disease. N Engl J Med 1993; 329: 332-42.

6. Grantham JJ. The etiology, pathogenesis and treatment of autosomal dominant polycystic kidney disease: recent advances. Am J Kid Dis 1996; 28: 788-803.

7. Mochizuki T, Wu G, Hayashi T, Xenophontos SL, Veldhuisen B, et al. PKD2, a gene for polycystic kidney disease that encodes an integral membrane proteine. Science 1996; 272: 1339-42.

8. Reeders ST. Multilocus polycystic kidney disease. Nature Gen 1992; 1: 235-7.

9. Tanner GA. Potassium citrate/citric acid intake improves renal function in rats with polycystic kidney disease.

10. J Am Soc Nephrol 1998; 9: 1242-8.

11. The European Polycystic Kidney Disease Consortium. The polycystic kidney disease 1 gene encodes a $14 \mathrm{~kb}$ transcript and lies within a duplicated region on chromosome 16. Cell 1994; 77: 881-94.

12. The International Polycystic Kidney Disease Consortium: Polycystic kidney disease: the complete structure of the PKD1 gene and its protein. Cell 1995; 81: 289-98.

13. Torres VE, Mujwid DK, Wilson DM, Holley KH. Renal cystic disease and ammoniagenesis in Han:SPRD rats.

14. J Am Soc Nephrol 1994; 5: 1193-1200.

15. Torres VE, Bengal RJ, Litwiller RD, Wilson DM. Aggravation of polycystic kidney disease in Han:SPRD rats by buthionine sulfoximine. J Am Soc Nephrol 1997; 8: 128391.

16. Torres VE. New insights into polycystic kidney disease and its treatment. Curr Opin Nephrol Hypertens 1998; 7: 159-64.

17. Viribay M, Hayashi T, Telleria D, Mochizuki T, et al. Novel stop and frameshifting mutation in the autosomal dominant polycysticc kidney disease 2 (PKD2) gene. Hum Genet 1997; 101: 229-34.

18. Xenophontos S, Constantinides R, Hayashi T, et al. A translation frameshift mutation induced by a cytosine in the polycystic kidney disease 2 gene. Hum Mol Genet 1997; 6: 949-52.

19. Yamaguchi T, Nagao S, Kasahara M, Takahashi H, Grantham JJ. Renal accumulation and escretion of cyclic adenosine monophosphate in a murine model of slowly progressive polycystic kidney disease. Am J Kid Dis 1997; 30: 703-09.

20. Ye M, Grant M, Sharma M, Elzinga L, Swan S, Torres VE, Grantham JJ. Cyst fluid from human autosomal dominant polycystic kidneys promotes cyst formation and expansion by renal epithelial cells in vitro. J Am Soc Nephrol 1992; 3: 984-94. 